\title{
Temporal Trends in the Incidence and Mortality of Skin Malignant Melanoma in China from 1990 to 2019
}

\author{
Ruhai Bai $\mathbb{D}^{1},{ }^{1}$ Hui Huang $\mathbb{D},{ }^{2}$ Minmin Li $\mathbb{D}^{3},{ }^{3}$ and Meng Chu $\mathbb{D}^{4}$ \\ ${ }^{1}$ School of Public Affairs, Nanjing University of Science and Technology, Nanjing 210094, Jiangsu, China \\ ${ }^{2}$ Department of Dermatology, The First Affiliated Hospital of Jinan University, Guangzhou 510632, Guangdong, China \\ ${ }^{3}$ Department of Infection Disease Control and Prevention, Shaanxi Provincial Center for Disease Control and Prevention, Xi'an, \\ Shaanxi 710054, China \\ ${ }^{4}$ School of Public Health, Health Science Center, Xi'an Jiaotong University, Xi'an 710049, Shaanxi, China \\ Correspondence should be addressed to Meng Chu; chumeng1110@163.com
}

Received 1 April 2021; Revised 1 August 2021; Accepted 15 August 2021; Published 24 August 2021

Academic Editor: San Lin You

Copyright (c) 2021 Ruhai Bai et al. This is an open access article distributed under the Creative Commons Attribution License, which permits unrestricted use, distribution, and reproduction in any medium, provided the original work is properly cited.

\begin{abstract}
Purpose. Skin malignant melanoma (SMM) is one of the fastest-growing cancers in China, with a poor prognosis, high invasiveness, and high mortality rate. The aim of this study was to determine the long-term trends in the incidence and mortality of SMM in China between 1990 and 2019. Patients and Methods. Incidence and mortality data were extracted from the Global Burden of Disease Study 2019 and were analyzed using an age-period-cohort framework. Results. The annual incidence net drifts were $3.523 \%$ (95\% confidence interval (CI): $3.318 \%$ to $3.728 \%$ ) and $3.779 \%$ (95\% CI: $3.585 \%$ to $3.974 \%$ ) for males and females, respectively, while the corresponding annual net drifts of mortality were $-0.754 \%$ (95\% CI: $-1.073 \%$ to $-0.435 \%$ ) and $-0.826 \%$ ( $95 \%$ CI: $-1.164 \%$ to $-0.487 \%$ ). The local drift from 1990 to 2019 was highest in males aged from 25 to 29 years. After controlling for period deviations in a single birth cohort, the SMM incidence and mortality increased exponentially with age for both sexes. Similar increasing monotonic trends were found for period and cohort effects on the incidence, while a declining trend was found for mortality. Conclusion. While the age-standardized mortality rate of SMM in China has decreased in both sexes over the past 30 years, the crude incidence rate, age-standardized incidence rate, and crude mortality rate have all increased. SMM may greatly threaten the health of the elderly in China due to the aging population. Appropriate changes should be made to raise the awareness, reduce the exposure to risk factors, and promote the early detection of SMM.
\end{abstract}

\section{Introduction}

Skin malignant melanoma (SMM) is the most aggressive and prevalent form of skin cancer that is characterized by a poor prognosis, frequent metastasis, and high mortality rate $[1,2]$. Melanoma only accounts for $1-2 \%$ of all cancer cases worldwide and less than $5 \%$ of skin cancers, but is responsible for around $95 \%$ of deaths due to skin cancer [3]. Melanoma can also appear early in life and is the solid tumor that causes the greatest number of potential life-years lost $[3,4]$.

The global incidence of melanoma has been increasing rapidly recently $[3,5]$, from 2.01 per 100,000 person-years in 1990 to 3.75 per 100,000 person-years in 2019 [6]. Although the incidence and mortality of SMM in China are lower than the global average, they have recently increased rapidly to become one of the fastest-growing cancers in China [7]. SMM in China is more aggressive and has higher mortality rates compared with European and American populations [8]. The 5-year survival rate of metastatic patients is lower than $20 \%$ [9].

Previous investigations of SMM in China have often focused on the risk factors, clinical features, and prognosis $[10,11]$. There have been some Chinese cancer-trend reports $[12,13]$ mentioning melanomas, but not specifically SMM. A few studies have compared the incidence and mortality between age groups, but these studies have not considered the impact of period and cohort effects. It is, therefore, 
necessary to conduct a comprehensive analysis of these limitations. This study aimed to use the 2019 Global Burden of Disease (GBD) data from 1990 to 2019 to determine the long-term trend in SMM incidence and mortality rates in China and use an age-period-cohort (APC) framework to analyze the corresponding effects on incidence and mortality rate between sexes. The present findings can provide guidance for etiology research of SMM morbidity and mortality, health resource allocation, and policy formulation to improve the prevention and treatment for high-risk groups.

\section{Materials and Methods}

2.1. Data Sources. The data analyzed in this study came from GBD 2019, which provides a systematic scientific assessment of published and publicly available data on incidence, prevalence, and mortality of disease and injury. GBD 2019 assesses the incidence, prevalence, mortality, years lived with disability, years of life lost, and disabilityadjusted life-years for 369 diseases and injuries for different age groups and sexes, covering 204 countries and territories around the world from 1990 to 2019 [6]. GBD 2019 data on China mainly come from two sources: [14] surveillance data from the China Disease Surveillance Points system (covers $24.3 \%$ of the total population of the country since 2013) and Vital Registration data (accounting for roughly $8 \%$ of the national population) collected by the Chinese Center for Disease Control and Prevention [15]. These two systems are well designed and would provide a nationally representative picture of diseases in China [15, 16]. For GBD data, many steps are taken to enhance the data quality, with bias reduced to some extent compared with that of research using raw data [17]. This study was reported according to the Reporting of studies Conducted using Observational Routinely-collected health Data Statement (RECORD) [18]. SMM is often determined by the following diagnostic codes: ICD-9 code 172-172.9 and ICD-10 codes C43-C43.9, D03-D03.9, D22-D23.9, and D48.5. SMM incidence and mortality in this study were standardized using the global age-standardized population of GBD 2019.

Data sources for the incidence and mortality rate of SMM can be explored using an online tool produced by the Institute for Health Metrics and Evaluation (http://ghdx. healthdata.org/gbd-2019). The GBD 2019 data are freely available to the world's researchers and policymakers, and it uses deidentified, aggregated data; therefore, a waiver of informed consent was reviewed and approved by the University of Washington Institutional Review Board.

2.2. Statistical Analyses. An APC analysis aims to assess how age, period, and cohort effects contribute to outcomes. The age effect represents the differing outcome risks associated with different age groups; the period effect represents outcome variations over time that influence all age groups simultaneously, and the cohort effect is associated with outcome changes across groups with the same birth years
[17]. The APC model was, therefore, used in this study to determine how age, period, and cohort effects influence the incidence and mortality of Chinese SMM patients.

The APC analysis was conducted by arranging incidence, mortality, and population data into consecutive 5-year periods from 1990 to 1994 (median 1992) to 2015-2019 (median 2017) and successive 5-year age intervals from 0-4 years to 90-94 years [19]. SMM incidence and mortality in patients aged $>95$ years were not considered in this study since those in this age group are divided into a single group in the GBD database. Parameters were estimated using the APC web tool (Biostatistics branch, National Cancer Institute, Bethesda, MD) [20]. Four functions were estimated using APC analysis. The first was net drift, corresponding to the overall log-linear trend in calendar periods and birth cohorts that indicate the overall annual percentage change. The second was local drift, corresponding to the age-groupspecific log-linear trend in calendar periods and birth cohorts, which indicates annual percentage changes for each age group. The third was longitudinal age curves, which indicate the fitted longitudinal age-specific rates in the reference cohort after adjusting for period deviations. The fourth was cohort (or period) relative risks (RRs), which represent the cohort (or period) RR adjusted for age and nonlinear period (or cohort) effects in a cohort (or period), against the reference cohort [17]. In the APC analysis, the median age group, period, and birth cohort were defined as the reference.

Wald chi-square tests were used for the significance tests of the estimating functions. All statistical tests were two sided and were considered statistically significant at $P<0.05$.

\section{Results}

3.1. Trends in SMM Incidence and Mortality Rates by Sex from 1990 to 2019. Figures 1(a) and 1(b) display the trends in crude incidence rate (CIR), age-standardized incidence rate (ASIR), crude mortality rate (CMR), and age-standardized mortality rate (ASMR) for SMM by sex for the period from 1990 to 2019. Figure 1(a) suggests that the CIR for SMM in China showed increasing trends for both sexes, from 0.318 to 1.161 and from 0.308 to 1.222 per 100,000 person-years for males and females, respectively. The ASIRs also showed increasing trends, from 0.416 to 0.932 and from 0.383 to 0.909 per 100,000 person-years for males and females, respectively. The ASIR for SMM increased by $124.04 \%$ and $137.34 \%$ in males and females, respectively. Figure 1(b) displays the changes in the SMM mortality rates of Chinese males and females and suggests that although the CMRs in both sexes showed increasing trends, from 0.237 to 0.345 and from 0.230 to 0.382 per 100,000 person-years for males and females, respectively, the ASMRs in both sexes had general decreasing trends, from 0.343 to 0.285 and from 0.309 to 0.271 per 100,000 person-years for males and females, respectively. It should be noted that the ASMRs for SMM increased slightly from 2006 to 2012 for both sexes. The ASMR for SMM reduced from 1990 to 2019 by $16.91 \%$ and $12.30 \%$ in males and females, respectively. 


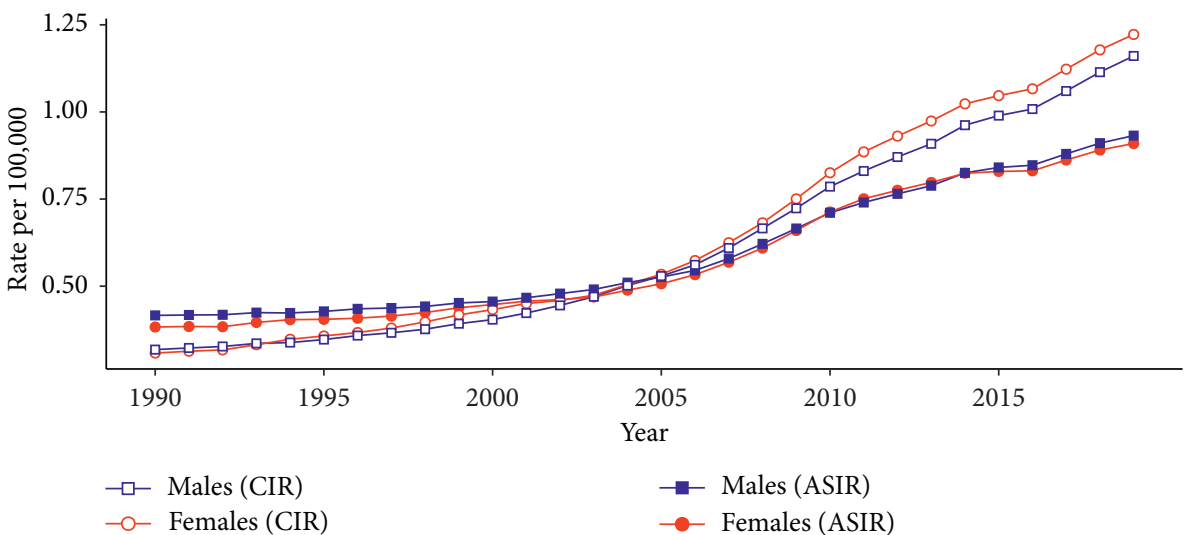

(a)

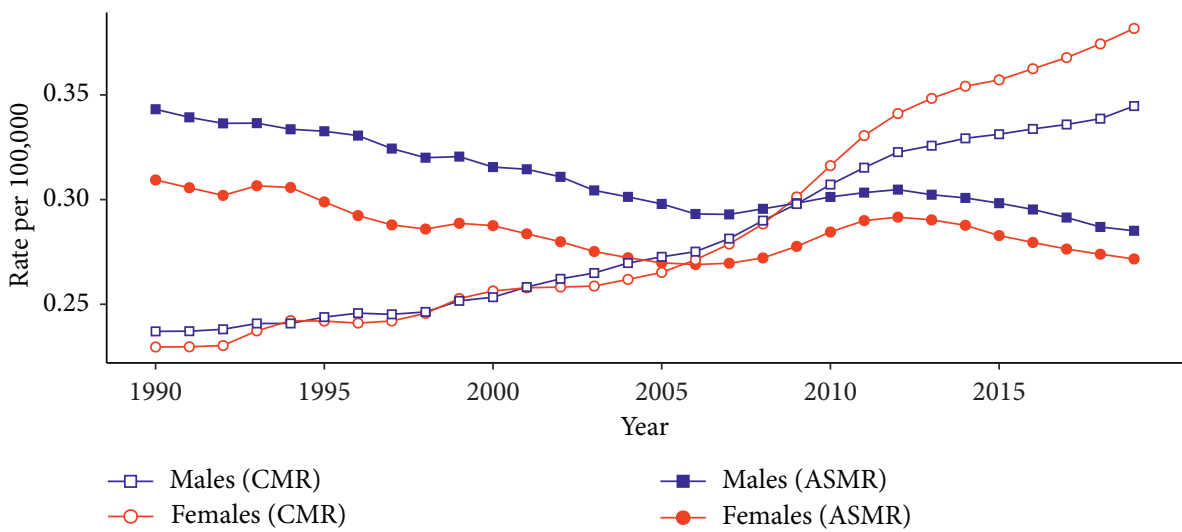

(b)

FIGURE 1: Trends in age-standardized and crude incidence and mortality rates per 100,000 person-years according to sex for skin malignant melanoma (SMM) in China from 1990 to 2019: (a) ASIR and CIR and (b) ASMR and CMR. The GBD 2019 global age-standard population data were analyzed. SMM: skin malignant melanoma; ASIR: age-standardized incidence rate; ASMR: age-standardized mortality rate; CIR: crude incidence rate; CMR: crude mortality rate; GBD: global burden of disease study.

\subsection{Local Drift with Net Drift Values for SMM Incidence and} Mortality Rates in China. Figure 2 displays the net drift (overall annual percentage change) and local drifts (annual percentage changes for each age group) for SMM incidence and mortality rates in China. The annual net drifts for SMM incidence were $3.523 \%$ (95\% confidence interval (CI): $3.318 \%$ to $3.728 \%$ ) and $3.779 \%$ (95\% CI: $3.585 \%$ to $3.974 \%$ ) for males and females, respectively. Figure 2(a) shows that the local drift values for SMM incidence were above 0 in all age groups for females $(P<0.05)$ and the highest in those aged 5-9 years at $4.705 \%$ (95\% CI, 3.569\% to 5.853\%). The local drift values for male SMM incidence were above 0 in all age groups $(P<0.05)$ except those aged 90-94 years and the highest in those aged $25-29$ years at $4.912 \%$ (95\% CI: $4.455 \%$ to $5.370 \%$ ). Overall, the SMM incidence increased more for females than for males in all age groups except for those aged $20-24$ and $35-39$ years. The annual percentage change was an increase of more than $4 \%$ in those aged 0-4 and 35-39 years for both sexes. The annual net drifts of SMM mortality were $-0.754 \%$ (95\% CI: $-1.073 \%$ to $-0.435 \%$ ) and $-0.826 \%$ (95\% CI: $-1.164 \%$ to $-0.487 \%$ ) for males and females, respectively, and the local drift values were below 0 in males aged 35-39 and 75-79 years and in females aged $20-24$ and $55-59$ years $(P<0.05)$.
3.3. Longitudinal Age Curves of SMM Incidence and Mortality by Sex in China. The longitudinal age curves of SMM incidence and mortality by sex are displayed in Figure 3. Males and females in the same birth cohort had a higher risk of SMM morbidity and mortality at ages of 0-4 and 90-94 years. Further estimations based on the longitudinal age curves indicated that both sexes exhibited exponential distributions. The incidence curves could be expressed as rate $=0.007 \times e 0.088 \times$ mean_age $\quad\left(R^{2}=0.994\right) \quad$ and rate $=0.008 \times e 0.086 \times$ mean_age $\left(R^{2}=0.985\right)$ for males and females, respectively, while the corresponding mortality rates were $0.014 \times e 0.062 \times$ mean_age $\left(R^{2}=0.955\right)$ and $0.015 \times e 0.062 \times$ mean_age $\left(R^{2}=0.988\right)$.

3.4. Period and Cohort RRs of SMM Incidence and Mortality Rate by Sex in China. Figure 4 displays the estimated period and cohort RRs for SMM incidence and mortality rate by sex. The period RRs of SMM incidence and mortality had similar patterns for both sexes (Figures 4(a) and 4(b)), with the incidence increasing significantly after the reference period (year 2000-2004) and the mortality decreasing after the reference period (year 2000-2004). The cohort RRs for 


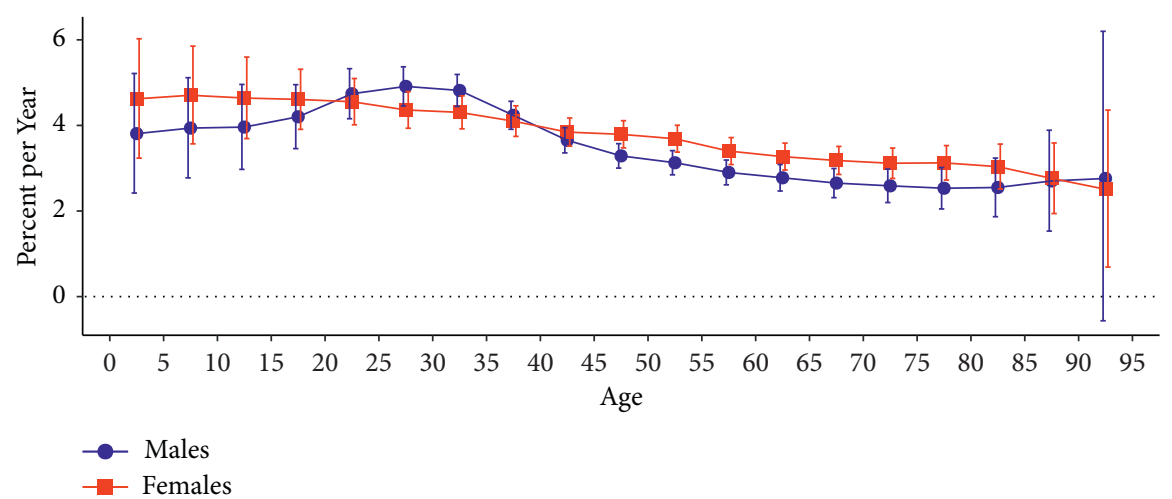

(a)

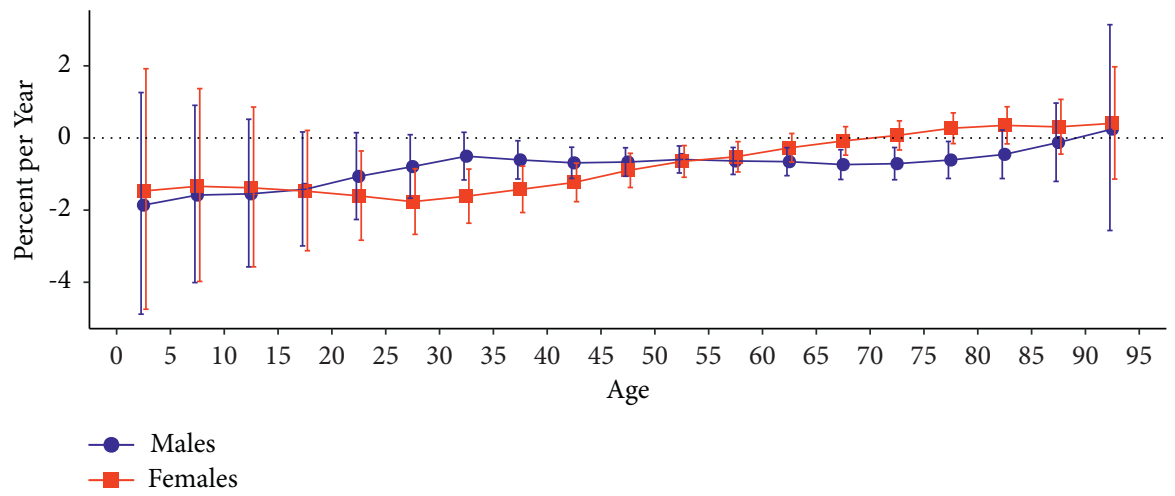

(b)

FIgURE 2: Local drift with net drift values for SMM incidence and mortality in China: (a) incidence and (b) mortality. Age-group-specific annual percentage change (local drift) with the overall annual percentage change (net drift) in SMM incidence and mortality rates and their corresponding 95\% CI are shown. SMM: skin malignant melanoma; CI: confidence interval.

incidence rate showed an overall increasing pattern from earlier to later birth cohorts for both sexes (Figure 5(a)), while the cohort RRs for the mortality rate had a decreasing pattern after the reference birth cohort (year 1955) for both sexes (Figure 5(b)).

\section{Discussion}

To our knowledge, this is the first study to investigate the long-term trends in the incidence and mortality of SMM in China and examine age-, period-, and cohort-specified effects using an APC framework. The present results indicate that the CIR and ASIR for SMM in Chinese males and females generally increased between 1990 and 2019, with increases in the SMM incidence rate for every age group except among those aged 90-94 years. Although the SMM CMR of Chinese males and females generally increased between 1990 and 2019, their SMM ASMR values showed overall decreasing trends among males aged 35-39 and 75-79 years and females aged $20-24$ and 55-59 years.

This study found a difference between the SMM CMR and ASMR, with the aging society considered to play an important role in this difference. China was considered an aging society from 2000, and the size of the elderly population is currently growing much more rapidly than any other age group [21]. The number of seniors aged $60-79$ and
80-94 years in China increased from 1953 to 2010 by about 3.5-6.6 times and 9.3-28.23 times, respectively. In 2010, people older than 60 and 65 years accounted for $13.3 \%$ and $8.9 \%$ of the total Chinese population, respectively [22], indicating rapid aging [21]. Age is an important demographic risk factor affecting the occurrence of and death due to SMM [23]. Based on the latest GBD 2019 data, our results indicate that SMM occurrence and mortality in the same birth cohort increase exponentially with age after adjusting for period deviation. Figure 3 displays the concentrated risk of SMM in China in the elderly stage, which may be related to the impaired immune response of the elderly to malignant tumors and infections [24]. Although the SMM ASMR has declined due to China's economic development [25], progression in medical technology $[2,26]$, and per-capita education level [26], the SMM CMR of the elderly has continued to increase due to increasing size of their population. Zeng and Wang [27] and Chen and Liu [28] estimated that by 2050, there will be 329 million people older than 65 years, accounting for $25 \%$ of the Chinese population, and the growth rate of people older than 80 years will be even greater, increasing from $0.9 \%$ in 2000 to $7.2 \%$ in 2050 . Moreover, there will be twice as many people older than 65 years in rural than in urban areas. Considering the current trends, we predict that the SMM CMR of Chinese males and females is likely to continue to increase in the future [24]. 


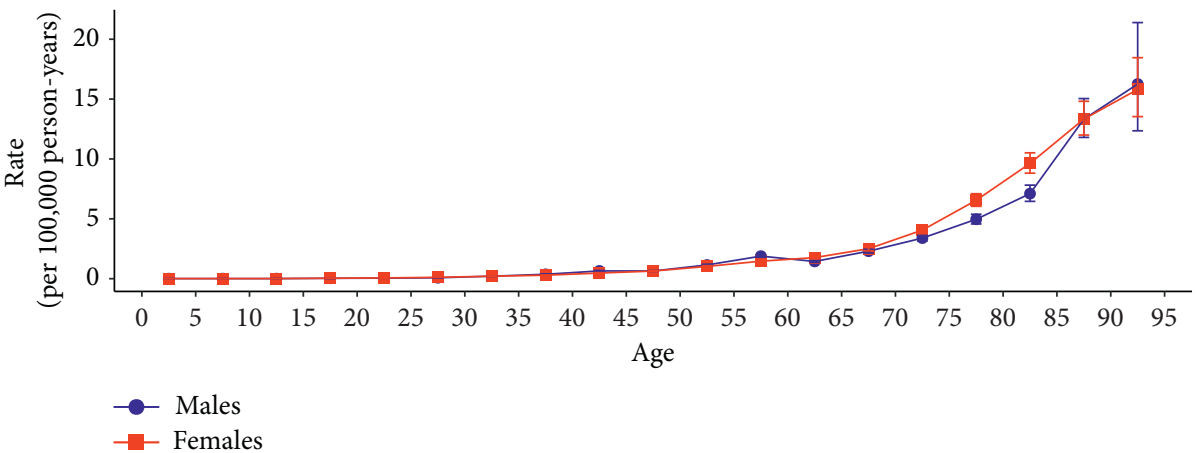

(a)

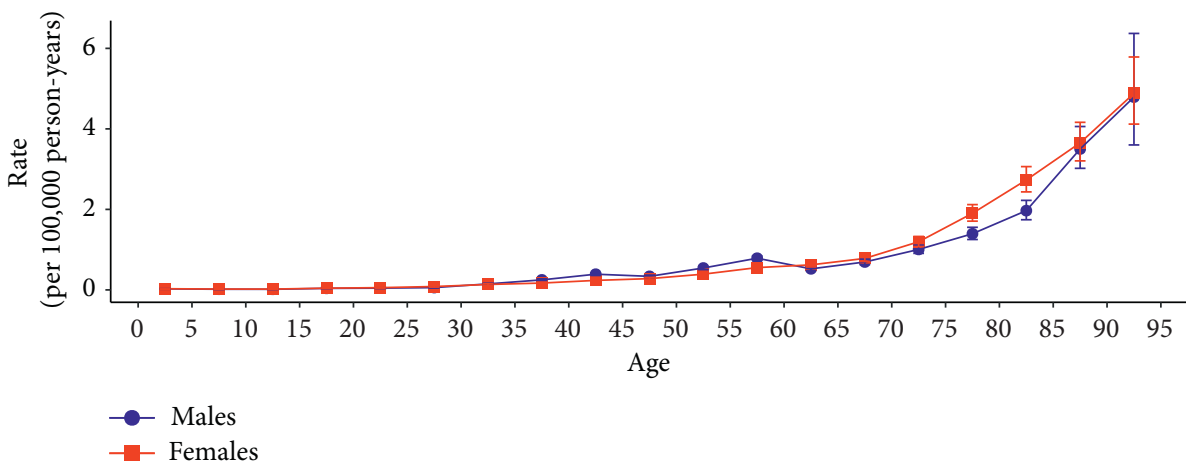

(b)

FIGURE 3: Longitudinal age curves of SMM incidence and mortality in China: (a) incidence and (b) mortality. Fitted longitudinal age-specific SMM incidence and mortality rates (per 100,000 person-years) and the corresponding 95\% CI (some of which are too narrow to see in the figure) are shown. SMM: skin malignant melanoma; CI: confidence interval.

It is noteworthy that the risk of melanoma is significantly higher in males than in females among Chinese people aged $20-40$ years, whereas it is significantly higher in females than in males among the United States population younger than 45 years $[23,29]$. Indoor tanning is a likely factor for the diverging trends in males and females younger than 50 years in the United States [29], while in China, indoor tanning is less popular. The risk might be higher in young Chinese males than females due to Chinese adult males tending to participate in more outdoor activities than females [30] and, hence, being exposed to more ultraviolet radiation [31]. Moreover, males are reportedly less aware of the hazards of excessive sun exposure and the correct protection measures (e.g., using a parasol) than females and are more likely to ignore public health education [31]. When outdoors, males are less likely to take appropriate sun protection measures [31], leading to the skin absorbing more ultraviolet radiation. Males are also more sensitive to ultraviolet radiation [32], which is responsible for $37 \%$ of melanomas in males but less than $9 \%$ of those in females [32]. All of these abovementioned factors may contribute to the morbidity risk for Chinese males aged 20-40 years being higher than that for females.

While period and cohort effects can be estimated separately as the period RR and cohort RR using certain restrictions, it is difficult to interpret them separately in the real world. This is because the period effect often influences age groups differently when it is applied to all age groups simultaneously, leading to the cohort effect [17]. It is, therefore, necessary to comprehensively discuss the possible reasons for the period and cohort effect trends. In this study, the period and cohort effects related to SMM incidence showed increasing trends, while the period and cohort effects for SMM mortality generally showed decreasing trends.

The increased SMM incidence may be related to the increase in the detection rate of melanomas in China. Compared with other cancers, malignant melanoma is characterized by skin localization, which allows for its early detection through noninvasive methods [33]. The detection and diagnosis rates of melanoma in China have recently been increasing with the developments in the economy and medical technology [34]. Simultaneously, the public's healthcare awareness has continuously progressed with improvements in education levels, as has the understanding of the early warning signs of diseases [26]. The number of people undergoing physical examinations has increased each year due to the rapid development of physical examination institutions and community service centers, which have also greatly increased the detection rate for the disease, therefore increasing the period and cohort RRs of the disease risk [35]. Although the increased diagnosis rate of early melanoma also leads to a rapid increase in the incidence, the Breslow thickness of the early lesions is usually shallow and the prognosis is often better [35]. This may also explain why the period and cohort RRs of incidence were found to be increasing while the mortality RRs were decreasing. 


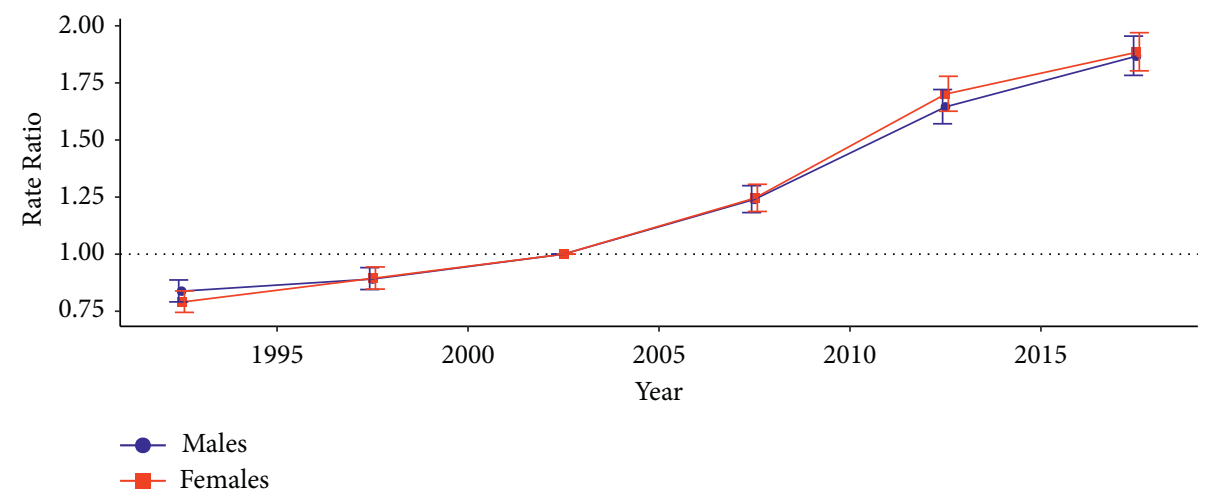

(a)

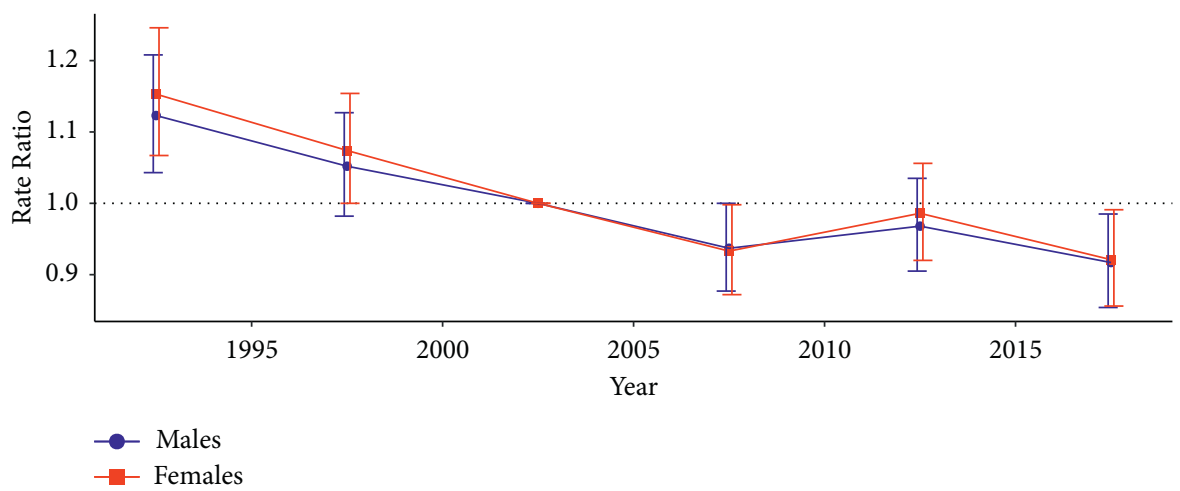

(b)

FIgURE 4: Period RRs of SMM incidence and mortality rates by sex in China: (a) incidence and (b) mortality. The RR of each period compared with the reference year (year 2000-2004) adjusted for age and nonlinear cohort effects and the corresponding 95\% CI are shown. RRs: relative risks; SMM: skin malignant melanoma; CI: confidence interval.

From a macroperspective, the increase in SMM risk is somewhat related to the increase in natural ultraviolet radiation. The ozone layer located in the stratosphere (10-50 km above sea level) absorbs all UVC and most UVB and UVA [36]. The destruction of the ozone layer increases human skin exposure to ultraviolet radiation [37]. Studies have indicated that a $1 \%$ reduction in the ozone layer is equivalent to a $1-2 \%$ increase in the melanoma mortality rate [37], and it has been predicted that there will be a significant thinning of the ozone layer by 2065 , which may lead to a very high incidence of skin cancer by 2100 [38]. Strong intermittent sun exposure can also significantly increase the melanoma risk [39]. Although previous studies have indicated that increases in melanoma incidence may also be related to increased use of indoor tanning equipment [29], the use of such equipment is less common in China, which, therefore, reduces its impact on the health of the population.

Primary prevention and early detection are crucial to addressing the health threat and economic burden of SMM [40]. We recommend that the general population should take measures to minimize their exposure to risk factors, such as avoiding exposure to intermittent high-dose sunlight as well as other types of ultraviolet radiation. Studies have indicated that although correctly using sunscreen can reduce the SMM incidence [40], traditional protection methods (e.g., wearing protective clothing and avoiding sun exposure or prolonged sunbathing) are more effective in reducing the melanoma risk [40, 41], and these measures cannot be replaced by sunscreen use [42]. To optimize protection from the damaging effects of the sun, we should, therefore, not just "slop on the sunscreen" but also "slip on a t-shirt, slap on a hat, seek shade, and slide on sunglasses." Importantly, these measures should be implemented together, not just one or some of them [42]. Regular screening is recommended for elderly people and high-risk groups who are susceptible to melanoma (e.g., people with fair skin, multiple atypical moles, or a family history of melanoma) with the purpose of diagnosing thin melanomas early and reducing the associated mortality [43]. Germany recommends regular skin cancer screening for people older than 35 years, while in the United States, it is not recommended [44]. However, some skin cancer prevention programs in the United States have had beneficial impacts on early diagnosis and treatment, and the survival rate at 5 years after a melanoma diagnosis increased from $50 \%$ in 1950 to $90 \%$ in 1990 [3].

There are notable limitations to our study. First, the Chinese SMM data used in this study are only macroestimates for the country. Like other APC analyses, there was an inevitability of ecological fallacy because interpreted population results are not necessarily valid for individuals. Related hypotheses from this study, therefore, still need further confirmation in future individual-based studies. Second, the limited 


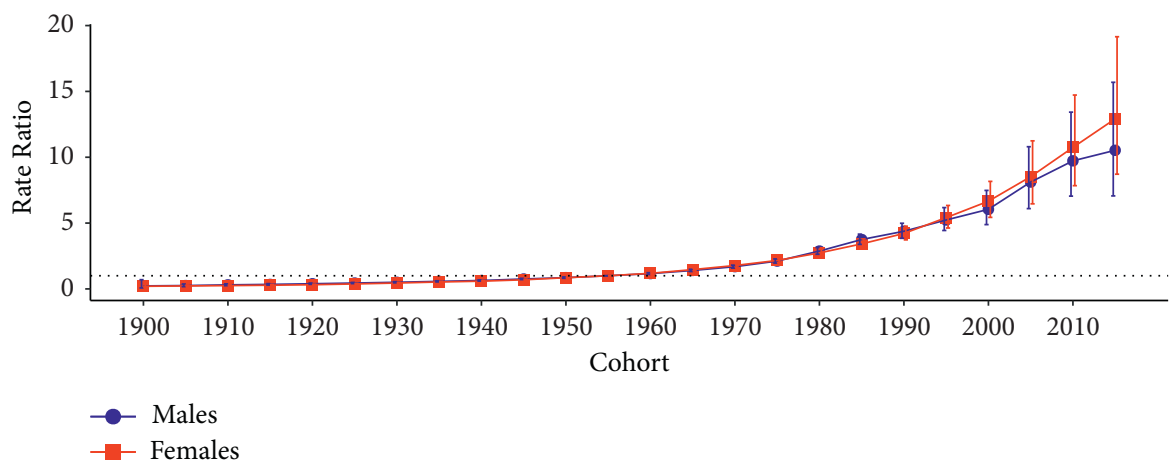

(a)

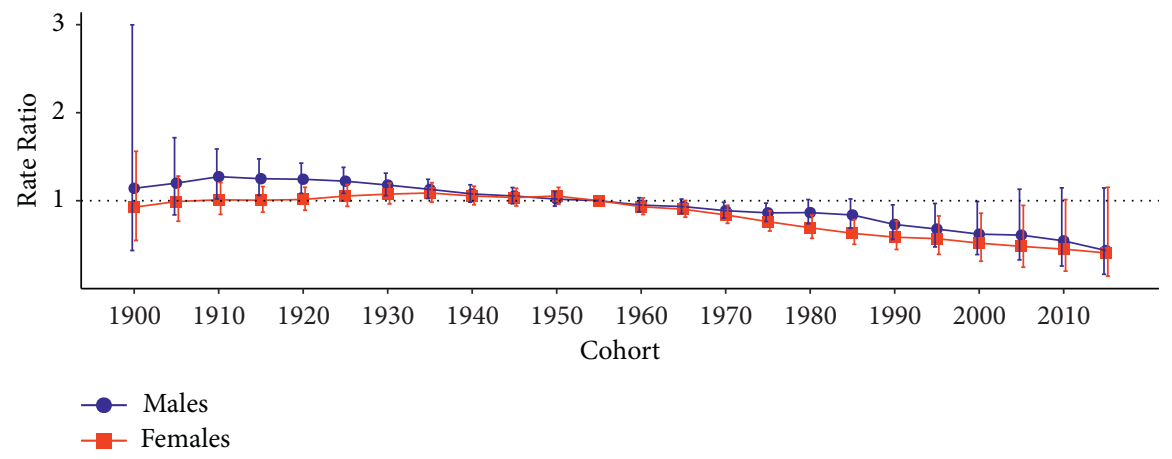

(b)

FIgURE 5: Cohort RRs of SMM incidence and mortality rate by sex in China: (a) incidence and (b) mortality. The RR of each cohort compared with the reference cohort (cohort 1955) adjusted for age and nonlinear period effects and the corresponding 95\% CI are shown. RRs: relative risks; SMM: skin malignant melanoma; CI: confidence interval.

data meant that we did not distinguish between urban and rural areas for the SMM incidence and mortality in China. Considering that China has a typical urban-rural dual structure, it is necessary to analyze the differences between urban and rural SMM cases in the future.

\section{Conclusions}

In summary, although the ASMR of SMM in Chinese males and females has shown a downward trend over the past 30 years, the CIR, ASIR, and CMR have all exhibited upward trends. Our APC analysis indicated that the SMM incidence and mortality rates in the same birth cohort increased exponentially with age for both sexes after controlling for period deviations. Considering that early SMM detection can effectively improve the overall survival and cure rates of patients [39], it is necessary to strengthen the knowledge that the Chinese public has of the harms caused by melanoma. The general population should reduce their exposure to ultraviolet radiation using techniques such as avoiding exposure to intermittent high-dose sunlight as well as other types of ultraviolet radiation and wearing protective clothing [39]. High-risk populations (e.g., severe sunburn, skin cancer, pigmented nevus, and chronic inflammation of the acral skin) [43] should be regularly screened and visit hospitals if necessary, with the aim of diagnosing melanoma with a low invasion depth early and, thereby, reducing mortality [43].

\author{
Abbreviations \\ APC: \\ Age-period-cohort \\ ASIR: Age-standardized incidence rate \\ ASMR: Age-standardized mortality rate \\ CI: $\quad$ Confidence interval \\ CIR: $\quad$ Crude incidence rate \\ CMR: Crude mortality rate \\ GBD: $\quad$ Global burden of disease \\ RECORD: Reporting of studies Conducted using \\ Observational Routinely-collected health Data \\ Statement \\ RRs: $\quad$ Relative risks \\ SMM: $\quad$ Skin malignant melanoma \\ UV: $\quad$ Ultraviolet.
}

\section{Data Availability}

The datasets generated and/or analyzed during the current study are available in the Institute for Health Metrics and Evaluation: http://ghdx.healthdata.org/gbd-2019.

\section{Ethical Approval}

The GBD 2019 data are freely available to the world's researchers and policymakers, and it uses deidentified, aggregated data; therefore, a waiver of informed consent was reviewed and approved by the University of Washington 
Institutional Review Board. Also, parameters were estimated using the APC web tool (https://analysistools.cancer.gov/ apc/) (Biostatistics branch, National Cancer Institute, Bethesda, MD).

\section{Conflicts of Interest}

The authors declare no conflicts of interest regarding the publication of this paper.

\section{Acknowledgments}

The authors are grateful to individuals who participated in the study. This work was financially supported by the Jiangsu Province Postdoctoral Research Funding Scheme (2021k548C).

\section{References}

[1] A. S. Alphonse and M. S. Starvin, "A novel and efficient approach for the classification of skin melanoma," Journal of Ambient Intelligence and Humanized Computing, pp. 1-25, 2020.

[2] A. Crispo, M. T. Corradin, E. Giulioni et al., Real Life Clinical Management and Survival in Cutaneous Malignant Melanoma: The Italian Clinical National Melanoma Registry (CNMR) Experience, Preprints, Basel, Switzerland, 2020.

[3] K. C. de Mélo, A. G. G. dos Santos, J. R. Amorim, and D. F. de Souza, "Temporal analysis of malignant skin melanoma mortality in Brazil: 2000 to 2016," Surgical \& Cosmetic Dermatology, vol. 11, 2019.

[4] S. Podlipnik, C. Carrera, A. Boada et al., "Incidence of melanoma in catalonia, Spain, is rapidly increasing in the elderly population. A multicentric cohort study," Journal of Clinical Medicine, vol. 9, no. 11, p. 3396, 2020.

[5] National Cancer Institute, SEER Cancer Stat Facts: Melanoma of the Skin, National Cancer Institute, Bethesda, MA, USA, 2020, https://seer.cancer.gov/statfacts/html/melan.html.

[6] GBD 2019 Diseases and Injuries Collaborators, "Global burden of 369 diseases and injuries in 204 countries and territories, 1990-2019: a systematic analysis for the Global Burden of Disease Study 2019," Lancet, vol. 396, no. 10258, pp. 1204-1222, 2020.

[7] H. Zhu, D. Dong, F. Li et al., "Clinicopathologic features and prognostic factors in patients with non-cutaneous malignant melanoma: a single-center retrospective study of 71 cases," International Journal of Dermatology, vol. 54, no. 12, pp. 1390-1395, 2015.

[8] J. Lv, B. Dai, Y. Kong, X. Shen, and J. Kong, “Acral melanoma in Chinese: a clinicopathological and prognostic study of 142 cases," Scientific Reports, vol. 6, no. 1, p. 31432, 2016.

[9] L. Chang, J. Pei, C. Li et al., "Incidence and metastasis of cutaneous malignant melanoma with respect to $\mathrm{ABO}$ blood groups: a case-controlled study in northeast of China," PLoS One, vol. 9, no. 2, Article ID e88096, 2014.

[10] X. Kang, Y. Zeng, J. Liang et al., "Aberrations and clinical significance of BRAF in malignant melanoma," Medicine, vol. 97, no. 1, Article ID e9509, 2018.

[11] M. Zhang and N. Zhang, "Clinical and prognostic factors in 98 patients with malignant melanoma in China," Journal of International Medical Research, vol. 45, no. 4, pp. 1369-1377, 2017.
[12] R.-M. Feng, Y.-N. Zong, S.-M. Cao, and R.-H. Xu, “Current cancer situation in China: good or bad news from the 2018 Global Cancer Statistics?" Cancer Communications, vol. 39, no. 1, p. 22, 2019.

[13] F. Bray, J. Ferlay, I. Soerjomataram, R. L. Siegel, L. A. Torre, and A. Jemal, "Global cancer statistics 2018: GLOBOCAN estimates of incidence and mortality worldwide for 36 cancers in 185 countries," CA: A Cancer Journal for Clinicians, vol. 68, no. 6, pp. 394-424, 2018.

[14] "Global burden of 369 diseases and injuries in 204 countries and territories, 1990-2019: a systematic analysis for the Global Burden of Disease Study 2019," Lancet, vol. 396, no. 10258, pp. 1204-1222, 2020.

[15] S. Liu, X. Wu, A. D. Lopez et al., "An integrated national mortality surveillance system for death registration and mortality surveillance, China," Bulletin of the World Health Organization, vol. 94, no. 1, pp. 46-57, 2016.

[16] F. Cui, L. Shen, L. Li et al., "Prevention of chronic hepatitis B after 3 decades of escalating vaccination policy, China," Emerging Infectious Diseases, vol. 23, no. 5, pp. 765-772, 2017.

[17] Z. Wang, S. Hu, S. Sang, L. Luo, and C. Yu, "Age-periodcohort analysis of stroke mortality in China," Stroke, vol. 48, no. 2, pp. 271-275, 2017.

[18] E. I. Benchimol, L. Smeeth, A. Guttmann et al., "The REporting of studies Conducted using Observational Routinely-collected health Data (RECORD) statement," PLoS Medicine, vol. 12, no. 10, Article ID e1001885, 2015.

[19] H. Huang, M. Li, H. Fan, and R. Bai, "Temporal trend of urolithiasis incidence in China: an age-period-cohort analysis," International Journal of General Medicine, vol. 14, pp. 2533-2539, 2021.

[20] P. S. Rosenberg, D. P. Check, and W. F. Anderson, "A web tool for age-period-cohort analysis of cancer incidence and mortality rates," Cancer Epidemiology Biomarkers \& Prevention, vol. 23, no. 11, pp. 2296-2302, 2014.

[21] X. Peng, "China's demographic history and future challenges,” Science, vol. 333, no. 6042, pp. 581-587, 2011.

[22] T. Liu, "Super-aging and social security for the most elderly in China," Zeitschrift für Gerontologie und Geriatrie, vol. 51, no. 1, pp. 105-112, 2018.

[23] E. A. L. Enninga, J. C. Moser, A. L. Weaver et al., "Survival of cutaneous melanoma based on sex, age, and stage in the United States, 1992-2011," Cancer Medicine, vol. 6, no. 10, pp. 2203-2212, 2017.

[24] S. K. Hui, W. Y. M. Tang, T. W. Wong et al., "Cutaneous melanoma: a population-based epidemiology report with 989 patients in Hong Kong," Clinical and Experimental Dermatology, vol. 32, no. 3, pp. 265-267, 2007.

[25] E. Crocetti, S. Mallone, T. E. Robsahm et al., "Survival of patients with skin melanoma in Europe increases further: results of the EUROCARE-5 study," European Journal of Cancer, vol. 51, no. 15, pp. 2179-2190, 2015.

[26] H. Eriksson, J. Lyth, E. Månsson-Brahme et al., "Low level of education is associated with later stage at diagnosis and reduced survival in cutaneous malignant melanoma: a nationwide population-based study in Sweden," European Journal of Cancer, vol. 49, no. 12, pp. 2705-2716, 2013.

[27] Y. Zeng and Z. Wang, "A policy analysis on challenges and opportunities of population/household aging in China," Journal of Population Ageing, vol. 7, no. 4, pp. 255-281, 2014.

[28] F. Chen and G. Liu, "Population aging in China," International Handbook of Population Aging, Springer, Berlin, Germany, pp. 157-172, 2009. 
[29] D. Lazovich, R. Isaksson Vogel, M. A. Weinstock, H. H. Nelson, R. L. Ahmed, and M. Berwick, "Association between indoor tanning and melanoma in younger men and women," JAMA Dermatology, vol. 152, no. 3, pp. 268-275, 2016.

[30] M. Su, Y.-y. Tan, Q.-m. Liu et al., “Association between perceived urban built environment attributes and leisure-time physical activity among adults in Hangzhou, China," Preventive Medicine, vol. 66, pp. 60-64, 2014.

[31] A. Streetly and H. Markowe, "Changing trends in the epidemiology of malignant melanoma: gender differences and their implications for public health," International Journal of Epidemiology, vol. 24, no. 5, pp. 897-907, 1995.

[32] F. Liu-Smith, A. M. Farhat, A. Arce et al., "Sex differences in the association of cutaneous melanoma incidence rates and geographic ultraviolet light exposure," Journal of the American Academy of Dermatology, vol. 76, no. 3, pp. 499-505, 2017.

[33] M. Rastrelli, S. Tropea, C. R. Rossi, and M. Alaibac, "Melanoma: epidemiology, risk factors, pathogenesis, diagnosis and classification," In vivo (Athens, Greece), vol. 28, no. 6, pp. 1005-1011, 2014.

[34] C. Carrera, M. A. Marchetti, S. W. Dusza et al., "Validity and reliability of dermoscopic criteria used to differentiate nevi from melanoma," JAMA Dermatology, vol. 152, no. 7, pp. 798-806, 2016.

[35] D. Moreno-Ramírez, T. Ojeda-Vila, J. J. Ríos-Martín et al., "The role of accessibility policies and other determinants of health care provision in the initial prognosis of malignant melanoma: a cross-sectional study," Journal of the American Academy of Dermatology, vol. 71, no. 3, pp. 507-515, 2014.

[36] R. Greinert, E. de Vries, F. Erdmann et al., "European code against cancer 4th edition: ultraviolet radiation and cancer," Cancer Epidemiology, vol. 39, no. Suppl 1, pp. S75-S83, 2015.

[37] H. E. Kanavy and M. R. Gerstenblith, "Ultraviolet radiation and melanoma," Seminars in Cutaneous Medicine and Surgery, vol. 30, no. 4, pp. 222-228, 2011.

[38] M. P. Chipperfield, S. S. Dhomse, W. Feng, R. L. McKenzie, G. J. M. Velders, and J. A. Pyle, "Quantifying the ozone and ultraviolet benefits already achieved by the Montreal Protocol," Nature Communications, vol. 6, no. 1, p. 7233, 2015.

[39] E. de Vries, F. I. Bray, J. W. W. Coebergh, and D. M. Parkin, "Changing epidemiology of malignant cutaneous melanoma in Europe 1953-1997: rising trends in incidence and mortality but recent stabilizations in western Europe and decreases in Scandinavia," International Journal of Cancer, vol. 107, no. 1, pp. 119-126, 2003.

[40] C. Carrera, J. A. Puig-Butillè, P. Aguilera et al., "Impact of sunscreens on preventing UVR-induced effects in nevi," JAMA Dermatology, vol. 149, no. 7, pp. 803-813, 2013.

[41] C. F. Garland, F. C. Garland, and E. D. Gorham, "Rising trends in melanoma an hypothesis concerning sunscreen effectiveness," Annals of Epidemiology, vol. 3, no. 1, pp. 103-110, 1993.

[42] D. L. Maslin, “Do suncreens protect us?” International Journal of Dermatology, vol. 53, no. 11, pp. 1319-1323, 2014.

[43] "Chinese guidelines for diagnosis and treatment of melanoma 2018 (English version)," Chinese Journal of Cancer Research, vol. 31, no. 4, pp. 578-585, 2019.

[44] D. Schadendorf, A. C. J. van Akkooi, C. Berking et al., "Melanoma," The Lancet, vol. 392, no. 10151, pp. 971-984, 2018. 